\title{
Die Birke: Botanik, medizinische Nutzung und kulturelle Bedeutung
}

\section{Barbara M. Wick \\ Zürich, Schweiz \\ Schlüsselwörter \\ Birke · Naturheilkunde · Botanik · Biomedizin · Volksmedizin . Forschung $\cdot$ Mythologie}

\section{Zusammenfassung}

Die Birke ist ein alter Pionierbaum (ca. 100 Millionen Jahre), der viel Licht benötigt, aber auch Licht spendet, die Allergiker irritiert, das Herz erfreut, beweglich erhält, Zauber abwehrt und Holz sowie Lebens- und Heilmittel zur Verfügung stellt. Dabei soll der Baum als Objekt (Botanik und allgemeine Verwendung) sowie dessen Rolle in der Medizin (Volksmedizin, Naturheilkunde, Biomedizin, Forschung) und in der Kultur (Mythologie und Rituale, Volksüberlieferung und Magie, Märchen und Legenden, Kunst, Symbolik) beleuchtet werden. Als besonderer Aspekt dieser ethnobotanischen Arbeit wird im Kapitel «Die Birke in der Symbolik» auf die medizinische Wirkung/ Verwendung im Kontext der Symbolik eingegangen.

(c) 2017 S. Karger GmbH, Freiburg

\section{Einleitung}

Die Birke ist ein alter Pionierbaum (ca. 100 Millionen Jahre), der viel Licht braucht, jedoch auch Licht spendet, die Allergiker irritiert, das Herz erfreut, beweglich erhält, Zauber abwehrt und Holz sowie Lebens- und Heilmittel zur Verfügung stellt.

Wer kennt diesen grazilen Baum nicht, der den Frühling ankündigt, Leichtigkeit und Freude vermittelt, seine Äste sanft bewegt und mit der weissen Rinde Orientierung bietet?

Vielen Völkern diente die Birke als unentbehrliche Lebensgrundlage und wurde entsprechend hoch geschätzt; in Russland gehört sie zum «nostalgischen Inventar».

Diese Arbeit basiert auf einer Projektarbeit des Zertifikatsstudiengangs (CAS) «Ethnobotanik und Ethnomedizin» der Universität Zürich, 2016 (Betreuerin: Dr. Caroline Weckerle, Institut für Systematische Botanik der Universität Zürich, Schweiz).

\section{Keywords \\ Birch · Naturopathy · Botany · Biomedicine · Folk medicine · \\ Pharmaceutical research $\cdot$ Mythology}

\section{Summary}

The Birch: Botany, Medical Use and Cultural Significance

The birch is an ancient pioneer tree (approximately 100 million years) which does not only require plenty of light but also gives light, irritates the allergy sufferers, delights the heart, keeps flexible, wards off evil, and provides timber, food and remedies. On this ethnobotanical journey the birch will be described as an object (botany and general use) as well as its role in medicine (folk medicine, naturopathy, biomedicine, pharmaceutical research) and in culture (mythology and rituals, popular tradition and magic, fairy tales and legends, art, symbolism). As a special aspect, the medical use/effect in the context of the symbolism of the birch will be focused on.

Eine Szene nördlich des Polarkreises: Unterwegs zum «Witch Memorial» (Hexenmahnmal) von Peter Zumthor und Louise Bourgeois im norwegischen Vardø sehe ich am Strassenrand drei Stühle vor einer Birke. Sie wirken arrangiert, doch weit und breit finden sich keine Hinweise auf Menschen (Abb. 1). Ich nutze die Gelegenheit, diese Stühle zu besetzen und bei der Birke zu verweilen.

Im Zuge dieser Übersichtsarbeit soll der Baum als Objekt (Botanik und allgemeine Verwendung) sowie dessen Rolle in der Medizin (Volksmedizin, Naturheilkunde, Biomedizin, Forschung) und in der Kultur (Mythologie und Rituale, Volksüberlieferung und Magie, Märchen und Legenden, Kunst, Symbolik) beleuchtet werden.

Als besonderer Aspekt dieser ethnobotanischen Arbeit wird im Kapitel «Die Birke in der Symbolik» auf die medizinische Wirkung/Verwendung im Kontext der Symbolik eingegangen.

\section{KARGER}

Fax +497614520714 Information@Karger.com www.karger.com

\section{(C) 2017 S. Karger GmbH, Freiburg}




\section{Die Birke in der Botanik und als Rohstoff}

\section{Botanik und Habitat}

Bei der Birke handelt es sich um einen der frühen Bäume; Fossilienfunde belegen eine Existenz schon vor dem Aussterben der Dinosaurier vor ca. 65,5 Millionen Jahren $[1$, S. 5]. Nach der letzten Eiszeit vor rund 12000 Jahren breitete sich der Baum in den kühlen und gemässigten Zonen der Nordhalbkugel aus [2].

Für viele Völker stellte die Birke über lange Zeit eine lebenswichtige Ressource dar und wurde dementsprechend geschätzt und verehrt.

\section{Gattung}

Die Gattung der Birke (Genus: Betula) gehört, wie die nah verwandte Erle (Alnus species), zur Familie der Birkengewächse (Betulaceae). Je nach Literatur werden weltweit ca. 40-60 Birkenarten gezählt [3, S. 150].

\section{Habitat}

Birken wachsen in klimatisch milden Zonen der Nordhemisphäre, grossteils in Waldgebieten des nördlichen Mittelmeerraums bis Asien, und finden sich auch in den USA sowie in Kanada, Grönland, Island, Alaska, Australien und Neuseeland. In Europa sind vorwiegend die weissrindige Hänge-Birke (Betula pendula Rотн) (Abb. 2) und die Moor-Birke (Betula pubescens Eнrн.) (Abb. 3) vertreten [3, S. 150].

\section{Standort}

Birken lassen sich je nach Umgebungsbedingungen an sehr verschiedenen Standorten (von feucht bis trocken) und in vielen Formen finden - von hohen schlanken Bäumen (bis zu 30 Metern) bis zu kleinen Tundrabüschen.

\section{Rinde}

Die Rinde ist glatt, harzig, weiss oder vielfarbig (viele Töne und Nuancen bis schwarz). Sie kann in zwei Schichten unterteilt werden: die äussere Schicht (Birkenkork) und darunter die eigentliche Rinde, die zusammen mit dem Holz aus dem Kambium hervorgeht. Die weisse Farbe ist auf das eingelagerte Triterpenderivat Betulin (modifiziertes, brennbares Harz) zurückzuführen [4]. Birken mit dunkler gefärbter Rinde sind geschichtlich älter, z.B. die Schwarz-Birke (Betula nigra L./USA) [1, S. 222] oder die Kupfer-Birke (Betula albosinensis BurkiLL/Asien) $[1$, S. 261].

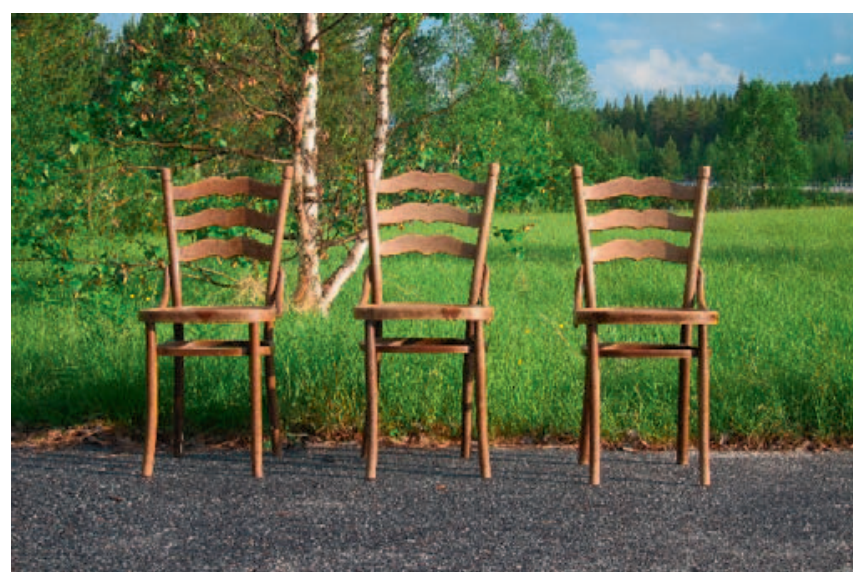

Abb. 1. Holzstühle vor Moor-Birke (Betula pubescens EHrH.), Teil 1, Norwegen (Copyright: Barbara M. Wick).

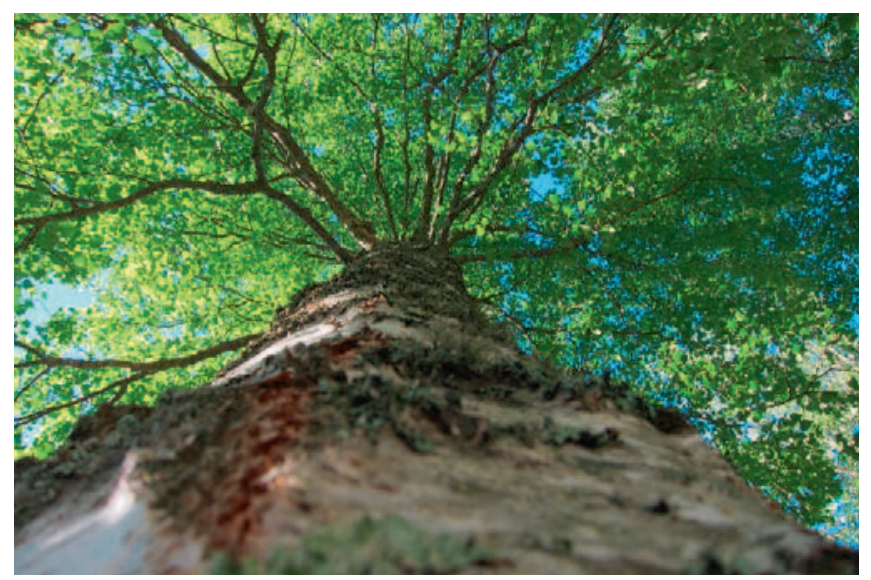

Abb. 2. Hänge-Birke (Betula pendula Rотн) im Sommer, Schweden (Copyright: Barbara M. Wick).

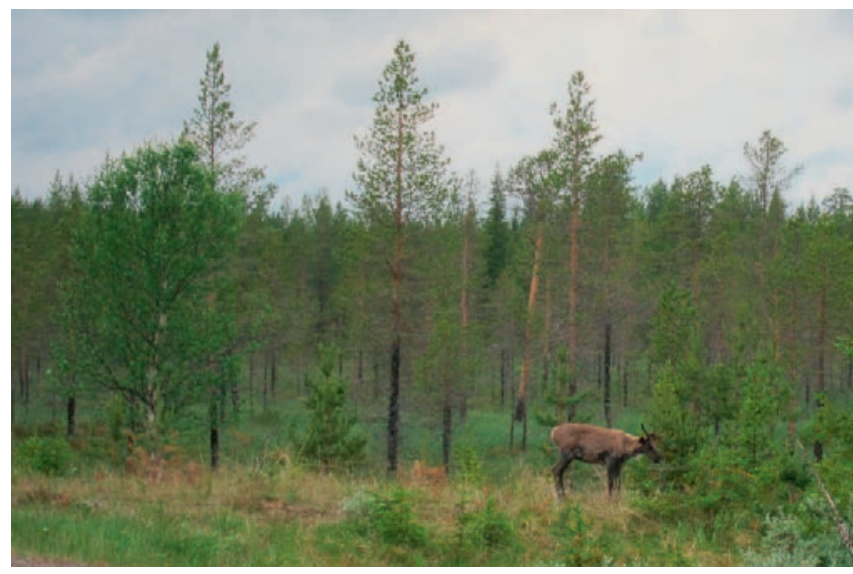

Abb. 3. Moor-Birke (Betula pubescens Eнrh.) und Rentier, Norwegen (Copyright: Barbara M. Wick).

\section{Blätter}

Die Birke hat wechselständige, zarte, kleine Blätter ohne Nebenblätter, die eiförmig bis dreieckig, mit gesägten Rändern und leicht haarigen Unterseiten (B. pendula ist unbehaart) sind. Im Frühjahr sind die Blätter klebrigharzig und balsamisch riechend. 


\section{Blüten}

Blütezeit ist von März bis Mai; die Birke ist einhäusig getrenntgeschlechtig. Die weiblichen Blütenstände sind grünliche, aufrechte Kätzchen; die männlichen Blütenstände sind gelblich-bräunliche, hängende Kätzchen.

Die Birkenpollen sind als starke Allergene bekannt. Die vielen, sehr kleinen Pollen (Blüte ab April) mit der je nach Windverhältnissen extremen Reichweite stellen einen bedeutenden Allergieauslöser dar und werden diesbezüglich für zunehmende Gesundheitsbelastungen verantwortlich gemacht. Globale Klimaveränderungen führen möglicherweise zu einer Zunahme und einem früheren Auftreten der Pollen; deshalb wurde in den USA schon diskutiert, alle Birken zu entfernen [5, S. 33].

\section{Früchte}

Die Reife der Früchte erfolgt von Juni bis August; hierbei werden Flügelfrüchte (Nüsschen mit beidseitigen Flügeln) gebildet.

\section{Inhaltsstoffe}

Saponine, Flavone, Triterpene, Tannine, Vitamin C, Xylit, Mineralstoffe, ätherische Öle, Gerb- und Bitterstoffe usw.

\section{Charakteristika}

Birken sind sehr anpassungsfähig, stellen wenige Ansprüche an den Boden und drängen sich als kreuzungsfreudige, kurzlebige Pionierpflanzen in Waldlücken, Ödland und Industriebrachen vor. Ihr grosser Lichthunger lässt sie zwischen schattentoleranteren Bäumen zurückweichen. Die wasserundurchlässige, luftgepolsterte, weisse Rinde schützt die Birke vor starker Sonneneinstrahlung und etabliert sie als winterhärtesten Laubbaum, der auch Minustemperaturen erträgt. Der weisse Farbstoff Betulin (Triterpenderivat) der weissrindigen Arten ist unter anderem für die Wasserundurchlässigkeit und den Schutz gegen Tierfrass verantwortlich.

\section{Soziales}

Die Birke gewährt Lebensraum für viele Tiere, z.B. für ca. 200-570 Insektenarten, für ca. 32 Vogelarten (z.B. Birkenzeisig [6]), für Mäuse (z.B. Waldbirkenmaus) und für Bienen. Auer- und Birkenhühnern dienen die Knospen als Winternahrung [7]. Die Birke lebt symbiotisch mit vielen verschiedenen Pilzen (z.B. Birken-Röhrling, Birken-Milchling, Fliegenpilz) und Misteln. Zu den Birkenschädlingen gehören z.B. der Birkenspinner und der Birkenporling [5, S. 99]. Junge Bäume sind besonders anfällig für Schäden durch Wildverbiss (Haarwild).

\section{Name und Namensherkunft}

Die Bezeichnung «Birke» (althochdeutsch «bircha») gehört zu den ältesten Baumnamen, bezieht sich auf die weisse, fein glänzende Rinde und geht auf den indogermanischen Wortstamm «bhereg» (hell schimmern, leuchten) zurück [8, S. 58].

Deutsch: Birke, Birkenbaum, Birkenmaie, Weiss-Birke, Silberbirke, Maienbirke, Warzenbirke, Rauchbirke, Harzbirke, Trauerbirke, Hänge-Birke, Marenquaste [3, S. 150]. Englisch: Birch (Tree), Birk, Birth. Französisch: Bouleau. Italienisch: Betulla. Spanisch: Abedul. Portugiesisch: Bétula.

\section{Die Birke in Europa}

Wenn wir in Europa von der Birke (Betula alba L.) sprechen, haben wir das Bild der zierlichen und eleganten Weiss- oder Hänge-Birke (syn. Silberbirke: B. alba L. sensu Coste, syn. B. pendula Roth, syn. Betula verrucosa EHRH.) mit dem schmalen Stamm, der charakteristischen, schimmernd weissen, sich schälenden Rinde, den feinen Ästen und den zarten, im Frühling fast durchscheinenden Blättern vor Augen - oder jenes der kleineren, knorrigeren Moor-Birke (B. alba L. sensu Rotн, syn. B. pubescens Eнrн.) (Abb. 3) [9, S. 158]. Diese Birken wachsen, abgesehen vom äussersten Süden, überall in Europa bis zu einer alpinen Höhe von ca. 1900 Meter ü.M.

Beiden Arten sind die relative Kurzlebigkeit (ca. 80-100 Jahre), die weisse Rinde, eine Wuchshöhe von durchschnittlich 10-25 Metern sowie flache Wurzeln, die bis 400 Liter Wasser pro Tag aufnehmen können, gemeinsam.

Die schnell wachsende Hänge-Birke zeichnet sich in den ersten paar Jahren durch nach oben gerichtete Äste aus, die bald zu hängen beginnen; nach ca. 50 Jahren erreicht sie bereits ihre maximale Höhe. Die Rinde schält sich in hauchdünnen Lagen, verliert mit zunehmendem Alter ihre weisse Farbe und wird von der Basis her borkig und schuppig. Hänge-Birken wachsen auf eher sauren, sandigen Böden und in trockenen und lichten Laub- und Nadelwäldern, während Moor-Birken einen feuchten Untergrund bevorzugen.

Die äussere Unterscheidung der beiden Arten in der Natur ist nicht einfach. Die Moor-Birke wächst langsamer und ist kleiner; die jungen Zweige sind im Gegensatz behaart und nie hängend, die Blätter sind kleiner und wohlriechend, und der Stamm bleibt auch im Alter weiss.

Neben diesen beiden Hauptvertretern existieren in Europa auch die Strauch-Birke (Betula humilis ScHRANK) und die Zwerg-Birke (Betula nana L.) [5, S. 22]. Diese nach der letzten Eiszeit als ursprünglich arktische Pflanze aus dem Norden emigrierte Zwerg-Birke findet sich in der Schweiz als bedrohter «Eremit» noch in Gais, bei Einsiedeln und im Jura [10, S. 108; 11]. 


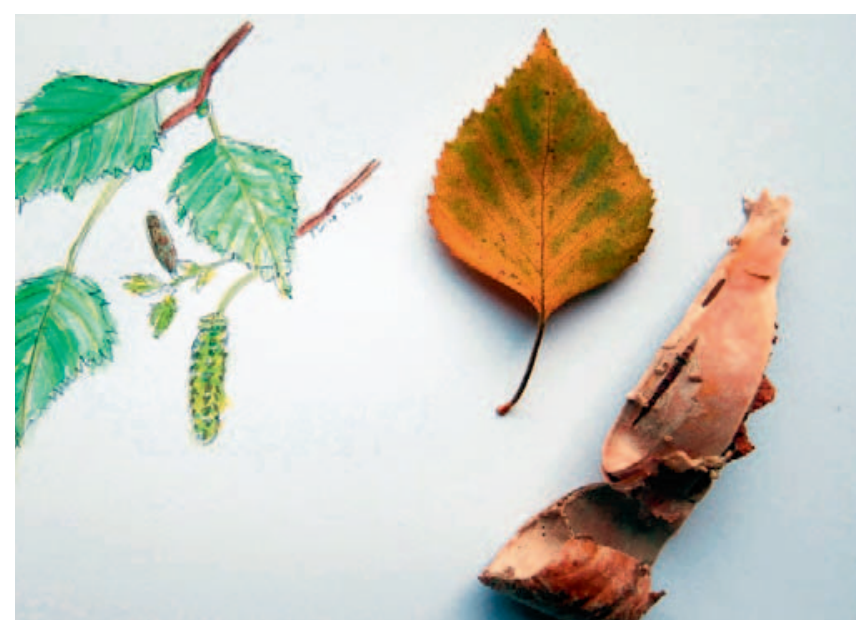

Abb. 4. Hänge-Birke (Betula pendula Rотн): Zeichnung, Herbstblatt und Rinde; Zürich (Copyright: Barbara M. Wick).

\section{Allgemeine Verwendung der Birke}

Die Birke ist Teil des Lebens, der Folklore und der Mythologie vieler Völker und essenziell im alltäglichen Leben.

\section{Holz, Äste und Blätter}

Seit alters findet das relativ harte und leichte Holz Verwendung für Zäune, Möbel und Furnier, Leitern, Baumaterial, Boots- und Flugzeugkonstruktionen («Fliegerbirken» [8, S. 59]), Musikinstrumente, Werkzeuge, Gerätschaften, Besen und Trinkgefässe sowie als Brenn- und Räucherholz, Fackeln, Kohle usw. Druckerschwärze bestand früher hauptsächlich aus Birkenruss. Den dekorativen Charakter der hellen Stämme nutzt man für die Innendekoration und in der Werbung.

Frische Birkenäste (Abb. 4) sind immer noch in der finnischen Sauna im Einsatz. Junge Blätter können als Salat, Suppe oder Spinat gegessen oder als Tee getrunken werden.

\section{Birkenrinde}

Birkenrinde (Abb. 5) diente schon früh als Schreibmaterial (Manuskripte auf Birkenrinde um $700 \mathrm{v}$. Chr. [5, S. 191]); im Ersten Weltkrieg waren in Russland Postkarten aus Birkenrinde gebräuchlich. Aus der Rinde werden teils heute noch Dachschindeln, Gefässe, Taschen, Schuhe, Bekleidung und Schmuck hergestellt.

Bei der Gletschermumie Ötzi (ca. 5300 Jahre alt) befanden sich zwei Behältnisse aus Birkenrinde [12].

Die Rinde lieferte bei gewissen indigenen Völkern auch einen Werkstoff für den Bau von Wigwams und Kanus und stellte verschiedene Heilmittel zur Verfügung. Ebenfalls diente ihnen die zucker- und ölhaltige Innenrinde in Zeiten der Not als Nahrungsmittel (Verarbeitung ge-

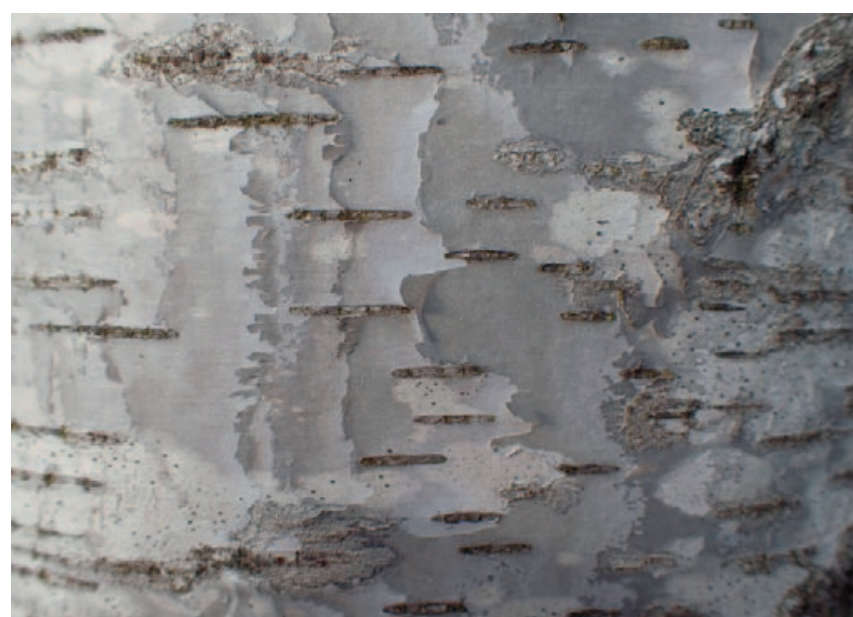

Abb. 5. Birkenrinde der Hänge-Birke (Betula pendula Rотн), Schweden (Copyright: Barbara M. Wick).

trockneter und gemahlener Innenrinde zu Brot, «Birkenspaghetti» oder Pfannkuchen [13, S. 26]).

Aus der Rinde lassen sich Gerbstoffe, Färbemittel und Feueranzünder fertigen. Birkenteer, die Vorstufe von Birkenpech, ist in Skandinavien zur Imprägnierung und zum Schutz von Holzbooten und -häusern im Einsatz. Birkenpech (durch trockene Destillation der Birkenrinde gewonnen) hilft beim Kitten und Abdichten. Das schwarze Birkenöl dient zur Lederkonservierung (Juchtenleder) und als Ausgangsstoff für Parfum (Russisch-Leder) oder für Tiere als Schutz vor Insekten.

\section{Birkensaft}

Pro Tag kann eine Birke im März insgesamt ca. 40-50 Liter Birkensaft liefern [14].

Aufgrund des hohen Zuckergehaltes eignet sich der Saft für Birkenwein und Birkenchampagner.

Der Birkensaft gilt als Haarwuchs- und Hautmittel und wird entsprechend in der kosmetischen Industrie verwendet bzw. als entschlackendes Mittel eingenommen.

Der Saft enthält Xylitol (Birkenzucker, 40\% weniger Kalorien als Zucker) und wird als Zuckeraustauschstoff eingesetzt [3, S. 158].

Neuerdings finden sich in der «Nordic Cuisine» wieder Rezepte mit Birkenrinde [15]. «Birch water» wird als Trendgetränk propagiert [16].

\section{Die Birke in der Medizin}

\section{Geschichte der medizinischen Verwendung}

Ältere Quellen zur medizinischen Anwendung der Birke finden sich bei der Heiligen Hildegard von Bingen (1098-1179) (Birkenrinde als Wundverschlussmittel [17, 


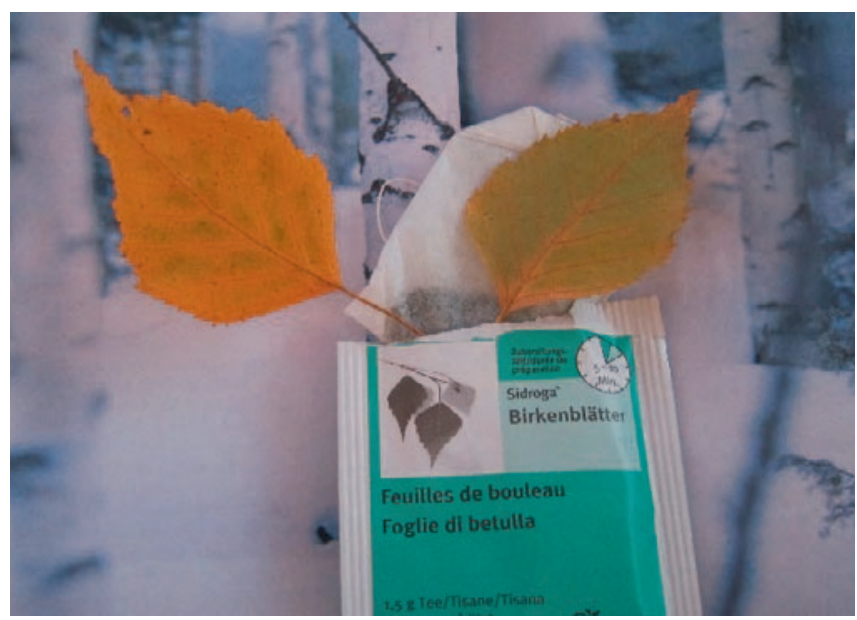

Abb. 6. Birkenblätter (Betulae folium) als Tee (Copyright: Barbara M. Wick).

S. 713]) und beim Arzt und Botaniker Matthiolus (15011577), der den Baum aufgrund seiner diuretischen Eigenschaften als «Nierenbaum» beschrieben hat.

Der englische Kräuterkundige Nicholas Culpeper benennt die Birke in seinem Buch «The complete Herbal and English Physician enlarged ...» aus dem Jahr 1653 als einen «Baum der Venus», dessen Saft Nieren- und Blasensteine auflösen könne [3, S. 156].

In Albrecht von Hallers «Histoire des Plantes Suisses ...» von 1791 wird Birkenöl aus verbrannter Rinde als Mittel gegen Hämorrhoiden und «même pour le cancer» aufgeführt [18, S. 256]. Gemäss Samuel Hahnemanns Apothekerlexikon von 1799 «dienen junge, frische Birkenblätter als Hausmittel, roh damit den Körper im Bette zu überdecken, um bei gichtischen und rheumatischen Fiebern Schweiss zu erregen oder sie, in einer erhitzten Pfanne erweicht, auf Wassergeschwülste zu legen oder mit einem Absude derselben Hautausschläge zu heilen» [19, S. 422]. In zwei Büchern (um 1900) aus dem Familiennachlass konnte die Autorin den Hinweis finden, dass die Rinde gegen Wechselfieber (Malaria tertiana) hilft [20, S. 65; 21, S. 35].

\section{Volksmedizin und Naturheilkunde}

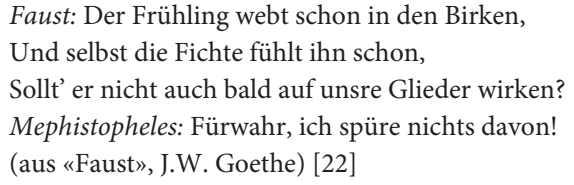

Faust: Der Frühling webt schon in den Birken, Und selbst die Fichte fühlt ihn schon,

Sollt' er nicht auch bald auf unsre Glieder wirken? Mephistopheles: Fürwahr, ich spüre nichts davon! (aus «Faust», J.W. Goethe) [22]

Die Birke ist ein altes Heil- und Hausmittel. Gemäss Alfred Sigrist erkannten frühere Heilkundige im gegen Wasser, Kälte und Schnee resistenten Baum besondere Beziehungen zu Erkältungskrankheiten und «Wasser»Krankheiten wie Nieren- und Blasenleiden, mangelnde Abwehrkraft gegenüber Kälte, Rheuma usw. [23, S. 64].
Der Tee aus den Blättern (Abb. 6) wird in erster Linie gegen Nieren- und Blasenaffektionen inklusive Stein- und Griessleiden angewendet, aber auch bei Ödemen, Gicht, Arthritis, Hautleiden, unterdrücktem Schweiss, Fieber, Infektionen, Fettleibigkeit und hohem Cholesterinspiegel. Ebenfalls werden gewisse antidepressive Eigenschaften postuliert [24, S. 65].

Ein Absud aus den Blättern dient als äussere Anwendung gegen Hautaffektionen und Fussschweiss.

Von Rheuma geplagte Glieder sollen noch im Jahr 1938 einige Tage lang mit frischen Blättern umhüllt werden [17, S. 718].

Birkensaft, früher ein Kraft- und Schönheitstrunk, wird immer noch als entschlackende und anregende Frühlingskur eingesetzt. Birkenwasser/-saft wird äusserlich gegen Hauterkrankungen und Schuppen sowie zur Wundheilungsförderung genutzt; in der Kosmetikindustrie erfolgt ein Einsatz als Haarwuchsmittel, gegen Schuppen und zur allgemeinen Hautpflege [13, S. 27].

Birkenöl (alkoholischer Extrakt der Birkenrinde) wirkt antiseptisch. Lotionen, Ölen und Salben mit Birkenextrakt werden Wirkungen gegen Hautinfektionen, kleine Hautverletzungen, Ausschläge und Rheuma zugeschrieben.

Birkenbäder (vor allem Rinde und Öl) wirken gegen Rückenschmerzen, Ischias, Rheuma und Hautprobleme.

Birkenkohle gilt als altbewährtes Mittel gegen Durchfall [13, S. 27].

In der Schweizer Naturheilkunde hat der Kräuterpfarrer Johann Künzle (1857-1945) Blätter, Knospen und Saft bei chronischem Rheuma und Blasenproblemen sowie als Wundheilmittel empfohlen [25, S. 327].

In den Schuhen getragene Birkenblätter sollen gegen rheumatische Leiden [3, S. 157] und Birkenreisig im Bett gegen Muskelkrämpfe helfen. Zur Heilung nervöser Leiden wurde Birkenholz als «lignum nervinum» eingesetzt; diese Verwendung datiert aus dem 17. Jahrhundert.

Verschiedenen indianischen Stämmen der USA war die Birke sehr wichtig; sie nutzten fast alle Teile der Birke zu Heilzwecken, z.B. die gekochte Birkenrinde gegen Hautverletzungen und -schwellungen, den Tee aus getrockneter Rinde und Blättern gegen Fieber, «Blutvergiftung», Erkältungen, Wurmbefall, Krämpfe, Schmerzen, Blähungen und Gicht sowie das Birkenöl gegen Anämie, Krätze, Tripper (der Siedler) usw. [26, S. 251].

\section{Moderne Phytotherapie, Biomedizin und Arzneimittel-} forschung

In der modernen Phytotherapie werden vorzugsweise die bitteren Blätter der Birke (Betulae folium; Inhaltsstoffe vor allem Flavonoide, Triterpene, Vitamin C) als wirksames Diuretikum eingesetzt, meistens in Form von Tee 
aus Blättern der Hänge- (B. pendula Roтн) oder MoorBirke (B. pubescens EHrH.). Die Flavonoide aus den Birkenblättern sollen über eine Hemmung des Angiotensinkonvertierenden Enzyms (ACE) und eine Hemmung des Abbaus von atrialem natriuretischem Peptid (ANP) eine verstärkte Natrium- und Wasserausscheidung induzieren [27].

Die diuretischen, nieren- und blasenanregenden Eigenschaften wirken unterstützend bei Harnwegsinfektionen, Gicht, Cellulitis, Ödemen, dermatologischen und rheumatischen Leiden, Arteriosklerose, Rachitis und Frühlingsmüdigkeit. Die Anregung der Gallensekretion kann einen leicht cholesterinsenkenden Effekt verursachen [5, S. 130].

In der Schweiz sind Birken-Präparate, ausser zur Desensibilisierung bei Allergien, in der Biomedizin wenig gebräuchlich und nicht kassenpflichtig. In der Komplementärmedizin existieren zahlreiche Präparate mit Birke, meist als Kombinationsmittel [28].

Im Januar 2016 wurde in Deutschland die Zulassung für ein Betulin-haltiges Medikament (Episalvan ${ }^{\circledR}$ ) zur Behandlung dermaler Wunden (Hautwunden und oberflächliche Verbrennungen Grad IIa bei Erwachsenen) erteilt. Das Gel enthält einen Trockenextrakt aus der Birkenrinde (Betulae cortex) von B. pendula Roтн [29]. Die Arzneimittelentwicklung erstreckte sich über 16 Jahre. Aufgrund einer galenischen Neuerung wurde dem Präparat der Innovationspreis für Phytotherapie verliehen [30]. Ende 2016 sind aufgrund der positiven Wirkungen weitere Studien mit Episalvan zur Behandlung der erblichen Hautkrankheit Epidermolysis bullosa geplant [31].

Gemäss der medizinischen Datenbank der National Libraries in Washington (www. pubmed.com [32]) existieren weltweit mehr als 1000 medizinisch-pharmakologische Studien zu Stoffen des Birkenkorks (vor allem zu den Triterpenen Betulin, Oleanol und Lupeol). Dabei werden verschiedene Wirkungen belegt: antibakteriell, antiviral, fungizid, antiinflammatorisch, zellwachstumshemmend und antitumoral, hepatoprotektiv, wundheilungsfördernd, Apoptose auslösend [33].

In einigen Versuchen lassen sich positive Wirkungen von Betulin auf den Stoffwechsel nachweisen (vor allem Senkung der Blutfettwerte und verbesserte Insulinempfindlichkeit) [34].

In vitro und in vivo zeigt Betulinsäure eine zytotoxische Aktivität gegen Melanome und aktinische Keratosen; in vitro wurde eine Aktivität gegen HIV, Herpes, Malaria [35] und Leishmaniose festgestellt [36].

Aufgrund der Hoffnung versprechenden Resultate und des Potenzials besteht weltweit ein grosses Interesse an der weiteren Erforschung medizinischer Anwendungen der Birke.
Abb. 7. Birke (Betula pendula RoTH) als «axis mundi» (Copyright: Barbara M. Wick).

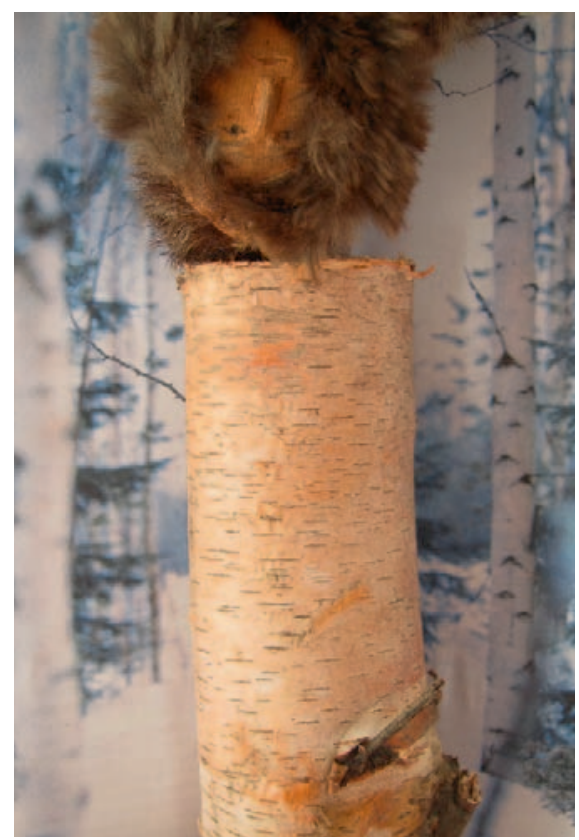

\section{Die Birke in der Kultur}

\section{Die Birke in Mythologie, Ritualen und Kulten}

Bäume spielten immer schon eine wichtige rituelle und magische Rolle in Naturreligionen. Bäumen wurden Opfergaben dargebracht, um Wohlstand von den Göttern zu erhalten. Bäume gehören zu den vielfältigsten und verbreitetsten Symbolen weltweit, z.B. der «Baum des Lebens» und der «Baum der Erkenntnis» in der Bibel.

Die Birke findet in der Antike keine schriftliche Erwähnung, da sie in südlichen Gefilden nicht vorgekommen ist.

Bei den Nordgermanen und Zentraleuropäern repräsentierte die Birke die Verbindung der Unterwelt der Toten und Dämonen mit der Oberwelt der Götter [37].

Die Birke war verschiedenen Gottheiten geweiht, z.B. der Mondgöttin (silbern schimmernde Rinde), in der nördlichen Mythologie der Göttin Freya und dem Kriegsund Donnergott Thor, ausserdem der Heiligen Brigid von Irland (451-525). Ihr Fest wird am Vorabend des Kirchenfestes «Lichtmess» am 2. Februar gefeiert, soll aber vor der Christianisierung dem keltischen Fest des Frühlingserwachens entsprochen haben und auf die keltische Göttin der Wiedergeburt des Feuers und der Vegetation, Brigid, zurückgehen. Ihr Name leitet sich wie jener der Birke ebenfalls vom indogermanischen Stamm «bhereg» (glänzen) ab [8, S. 61].

In Russland pflanzten Bauern zu Pfingsten Birkenzweige als Symbol des Frühlingserwachens.

In Sibirien gilt die Birke als wichtiges Element der Reinigung und Initiation der Schamanen: Ein ausgewählter 
Baum wird in der Jurte, mit der Wurzel in der Feuerstelle, platziert. Die Krone dieses «kosmischen Pfeilers» oder «axis mundi» (Abb. 7) ragt aus dem Rauchabzug. Die in Trance versetzende Trommel stammt aus dem Holz dieser Birke. Man glaubt, dass auf einer schamanischen Reise durch die obere Öffnung der Jurte vom Kosmos zum Polarstern gelangt werden kann. Die Birke symbolisiert den Pfad der Himmelsenergie zum Menschen und jenen des menschlichen Verlangens zum Himmel [3, S. 152].

Es ist möglich, dass der mit der Birke in Symbiose wachsende Fliegenpilz sowohl die Trance-Reisen als auch die Auswahl des «kosmischen Baumes» begünstigt [8, S. 61].

Bei gewissen indigenen Völkern war es Brauch, Tote vor dem Verbrennen rituell in Birkenrinde einzuwickeln, da Birkenrinde als heiliges Geschenk des Geistes und Kulturheros Nanabozho angesehen wurde [38].

\section{Die Birke in der Volksüberlieferung und Magie}

\section{Die Birke als Maibaum}

Wie in Belgien und den Niederlanden ist der Brauch des Mai- oder Liebesbaums auch heute noch in Teilen Deutschlands und der Schweiz bekannt. Dabei wird in der Nacht zum 1. Mai ein junger Baum als Zeichen der Zuneigung und Liebe vor die Tür einer auserwählten jungen Frau gestellt. Die Birke als erster blühender Baum des Frühlings ist besonders beliebt («Liebesmaie») und verkörpert mit ihren feinen Zweigen Schönheit und Güte. Der einer jungen Frau gewidmete Maibaum soll einen Monat stehen bleiben, sonst wird dies als Zeichen der Zurückweisung gewertet [39].

\section{Die Birke als «Lebensrute»}

Gemäss Tradition der keltischen Druiden sollen Schläge mit einer Birkenrute die dem Baum innewohnende Lebenskraft auf das geschlagene Tier oder die geschlagene Person übertragen; diese Assoziation mit einem Fruchtbarkeitszauber soll bis in die Antike zurückgehen.

In einigen Gegenden Europas wurde das Vieh nach der Überwinterung mit Birkenzweigen auf die Weide getrieben. Ebenfalls sollten gelegentliche Schläge des Viehs mit einer Birkenpeitsche der Gesunderhaltung und Fruchtbarkeit dienen. Auch für den Menschen wurde das Prinzip dieser Übertragung von Kraft angewendet [3, S. 153].

\section{Die Birke als Schutz des Viehs und der Ernte}

Gemäss Volksüberlieferung überträgt die Birke nicht nur Kraft und Gesundheit, sondern kann auch Unheil abwenden und gegen Ungeziefer eingesetzt werden. So soll ein Kuhstall von Läusen und Schädlingen verschont bleiben, wenn man ihn zu Glockengeläut mit einem Bir- kenbesen, dessen Birkenreisig an Weihnachten geschlagen wurde, gereinigt hatte [3, S. 154].

Der Brauch, in der Stube frische Birkenzweige gegen die Fliegenplage aufzuhängen, wurde von der Autorin in den 1980er-Jahren in einem Appenzeller Bauernhaus beobachtet. Die Fliegen würden den Geruch nicht mögen, sagte man.

Die Birke als Schutz gegen Hexen und Unheil sowie als Züchtigungsmittel

Birkenzweigen wird zugeschrieben, Hexen und böse Geister fernhalten zu können. Dazu sind unzählige Rituale belegt, wie z.B. in Schweden in der Walpurgisnacht Birkenzweige an die Stalltüre zu hängen, eine junge Birke vor dem Hauseingang als Schutz gegen das Böse zu pflanzen [3, S. 155] oder die Grenzen eines Grundstücks mit Birkenzweigen abzuklopfen.

Vielerorts wurden Wiegen traditionellerweise aus Birkenholz angefertigt; dies wohl nicht nur als Schutz, sondern auch als Symbol des neuen Lebens.

Lonicerus (Adam Lonitzer, 1528-1586), ein deutscher Arzt, beschreibt die Birkenrute in seinem «Kräuter-Buch» als Züchtigungsmittel zur Erziehung störrischer Kinder: «O du gute Birkenruth, du machst die ungehorsamen Kinder gut!» [40, S. 94].

\section{Die Birke in der magischen Heilung}

Krankheiten können auf viele Arten von Menschen auf Bäume übertragen werden.

Ein Gichtgeplagter soll sich vor Sonnenaufgang leise einer Birke nähern, diese schütteln und seine Krankheit mit einem Spruch an den Baum abgeben: «Birkenbaum, ich schüttle dich, 77erlei Gichten quälen mich, Solang sollen sie in dir sein verbunden, Bis meine 77erlei Gichten verschwunden» (1928) [3, S. 156].

Das Auflegen eines für die Entfernung von Spinnenweben benutzten Birkenbesens auf einer kranken Stelle des Körpers galt als heilsam. Dem Heiligen Sigismund einen Birkenbesen zu stiften, half gegen Bettnässen [3, S. 156].

\section{Die Birke in Märchen und Legenden}

Märchen, in denen Birken figurieren, existieren überall, vor allem aber in Finnland, Russland, Litauen und Polen. Bei den Gebrüdern Grimm taucht die Birke im Märchen «Die zwei Brüder» auf.

Offenbar tat sich die Christenheit schwer mit der heidnischen Verehrung der Birke, sodass viele christliche Legenden die Birke als Baum des Bösen darstellten.

Gemäss einer schwedischen Sage sind die Birkenzweige zur Geisselung von Christus gebraucht worden; zur 
Abb. 8. Birken in gelber Erde (Copyright: Barbara M. Wick).

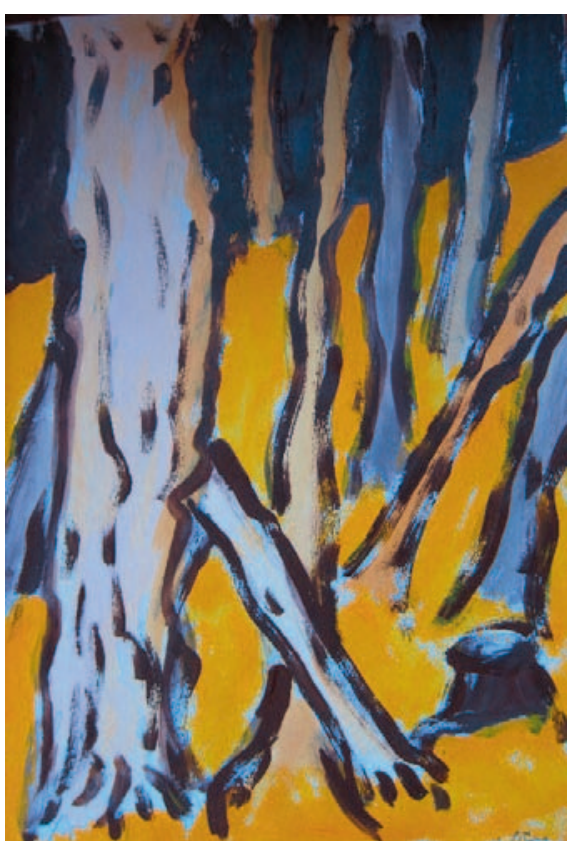

Abb. 9. Birke (Betula pendula Rотн) in Schweden zur Sommersonnenwende (Copyright: Barbara M. Wick).

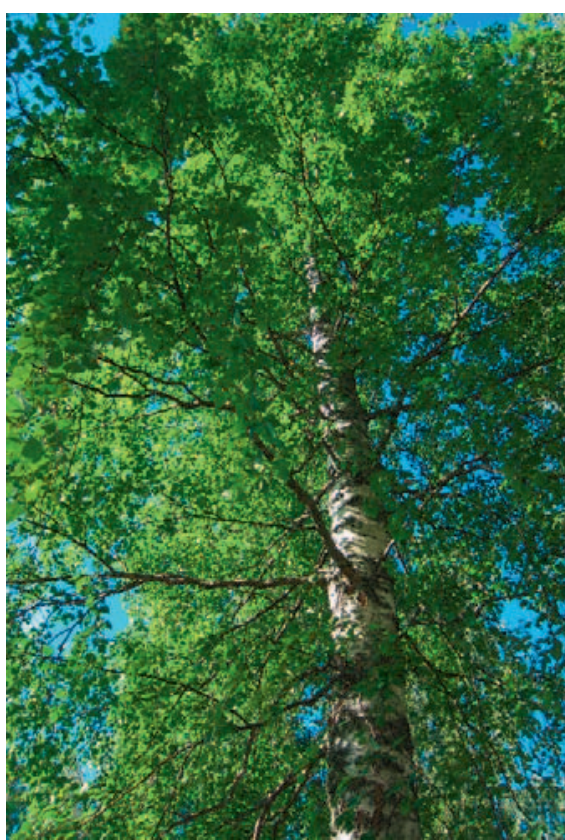

Strafe wurde der Baum zu hängenden Zweigen («weeping birch») verdammt [3, S. 155].

\section{Die Birke in der Kunst}

Die strahlenden, eleganten und lebendigen Bäume beleben düstere, karge und melancholische Gegenden und ziehen den Blick auf sich.

Es ist naheliegend, dass die Birke vor allem bei nördlichen Völkern in der Literatur und Dichtung, Musik, Malerei (Abb. 8), Fotografie, Film und Gebrauchskunst oft vorkommt.

Der sowjetische Filmemacher Andrej Tarkowskij (1932-1986), bekannt für seine eigenwillige, metaphorische Bilderwelt, setzte die Birke oft ein:

«Der Birkenwald ist ein gutes Beispiel für die Ambivalenz der Bilder Tarkowskijs. Einerseits ist bekannt, dass die Russen - wie auch die Polen - ein geradezu zärtliches Verhältnis zu den Birken haben, diesen wunderbar lichten Bäumen, die die Melancholie der osteuropäischen Landschaft aufhellen, aber gleichzeitig mit ihren feinen, traurig hängenden Zweigen in diese Melancholie einstimmen. In Nostalghia hören wir von dem Musiker Sosnofskij, dass es ihn schier umbrachte, seine russische Heimat mit den geliebten Birken nicht mehr wieder sehen zu können. Andererseits meinte Tarkowskij, dass dieser Birkenhain so etwas wie den 〈Hauch der schwarzen Pest〉 heraufbeschwor» [41].

In der Landesausstellung Expo.02 wurden im «Garten der Gewalt» unter anderen Bäumen viele regelmässig aufgestellte Birken inszeniert [42]. Heute lassen sich diese als «Birkenhof» im Innenhof eines Hotels in Zürich betrachten [43].

\section{Die Birke in der Symbolik}

\section{Die Birke in der Symbolik allgemein}

- Die Birke steht als Symbol des Frühlings, der Jugend, der Wiedergeburt und Erneuerung, der Fruchtbarkeit, der Reinheit, des Lichtes, der Liebe und der Freude. Sie verkörpert im Lebenskreislauf Wachsen und Entstehen. Die Birke ist mit dem Lebendigen verbunden und ein Symbol des Schutzes. Besonders wichtig ist sie in nord- und osteuropäischen Ländern, wo sie als erstblühender Baum den Frühling ankündigt.

- Die Birke wurde oft als Mädchen oder junge Frau gesehen, und wäre die Birke eine Nymphe, würde sie möglicherweise einer der Grazien Botticellis ähnlich sehen.

- Kulturgeschichtlich stellt die Birke die «Lebensrute», die Mensch und Tier gesund und stark erhält und vor Unheil schützt [3, S. 152].

- Bei Teilen des sibirischen Volkes gilt die Birke immer noch als wichtigster heiliger Baum und als Symbol für die Achse der Welt (axis mundi).

- Der Tag der Birke wird am 24. Juni, zur Zeit der Sommersonnenwende, gefeiert (Abb. 9).

- In der Blumensprache stehen Birken für Bescheidenheit und Anmut [3, S. 153].

\section{Medizinische Wirkung/Verwendung der Birke im Kontext der Symbolik}

Im Verlauf der Recherche zu botanischen, medizinischen und kulturellen Aspekten der Birke stellte sich für die Autorin als Ärztin die Frage, inwieweit symbolische 
Tab. 1. Medizinische Wirkung/Verwendung und Symbolik der Birke

\begin{tabular}{ll}
\hline $\begin{array}{l}\text { Medizinische Wirkung/Verwendung (Betula sp.) } \\
\text { Blätter (Tee/ Absud): diuretisch, säfteregulierend, } \\
\text { hautreinigend; gegen Fieber, Infektionen, } \\
\text { rheumatische Leiden, Arthritis und Gicht, }\end{array}$ & $\begin{array}{l}\text { Frühling, Jugend, } \\
\text { Erneuerung, } \\
\text { Wiedergeburt }\end{array}$ \\
Harnwegsinfektionen & \\
Räuchermittel gegen Infektionen \\
Saft: anregend (Niere, Blase, Leber). Frühlingskur. \\
$\quad$ Haarwuchsmittel, Kosmetika (Hautpflege und \\
-schutz, bei Hautunreinheiten und Cellulitis) \\
Betulin: Wundheilmittel (Hautdefekte, Verbren- \\
nungen) \\
Antibakterielle, antivirale und antitumorale Eigen- \\
schaften (z.B. aktinische Keratosen und Melanom). \\
Apoptose auslösend.
\end{tabular}

Blätter (Tee/Absud): gegen Hautausschläge, Reinheit Harnwegsinfektionen

Rinde (Absud): gegen Hautleiden, Krätze (indianische Medizin)

Holz (Kohle): gegen Durchfall

Betulin: stoffwechselanregend, cholesterinsenkend. Antiinflammatorische Wirkung (z.B. bei Psoriasis)

\begin{tabular}{ll}
\hline Blätter (Tee): mögliche antidepressive Wirkung & Freude, Licht \\
\hline Rinde: als Hautpflaster (indianische Medizin) & Schutz \\
Betulin: antibakterielle, antivirale, fungizide und & \\
$\quad$ hepatoprotektive Wirkung; Wirkung gegen & \\
$\quad$ Malaria, Herpes, HIV, Hepatitis, Leishmaniose & \\
\hline
\end{tabular}

Zuschreibungen die medizinische Verwendung von Birkenpräparaten begründet haben bzw. begründen könnten.

- Ist die als reinigend und verjüngend beschriebene Wirkung der Birke zufällig oder wird sie dadurch ihrer $\mathrm{Zu}$ schreibung als Symbol des Frühlings und der Erneuerung gerecht?

- Wird die Birke ihrer Zuschreibung als Symbol des Frühlings und der Erneuerung gerecht?

- Wird der antirheumatische Effekt im Zusammenhang mit der eleganten Beweglichkeit der Birke postuliert?

- Regt die weisse, sich schälende Rinde in Anlehnung an die Assoziation mit der menschlichen Haut zur Verwendung als Dermatologikum an?

Diesbezügliche Antworten und Andeutungen sind der Autorin nur in der Volksmedizin und Volksüberlieferung begegnet. In medizinischen oder ethnobotanischen Studien und Arbeiten klingen solche Verknüpfungen kaum oder gar nicht an.

Zusammenfassend werden in Tabelle 1 die medizinische Wirkung/Verwendung (Hausmittel, Volksmedizin, Biomedizin, Arzneimittelforschung, möglicher Einsatz in der Zukunft) gegenüber der Symbolik der Birke aufgeführt. Dies ist eine arbiträre Zuteilung, aus der eine gewisse Übereinstimmung zwischen medizinischer Wirkung/ Verwendung und Symbolik ersichtlich wird.

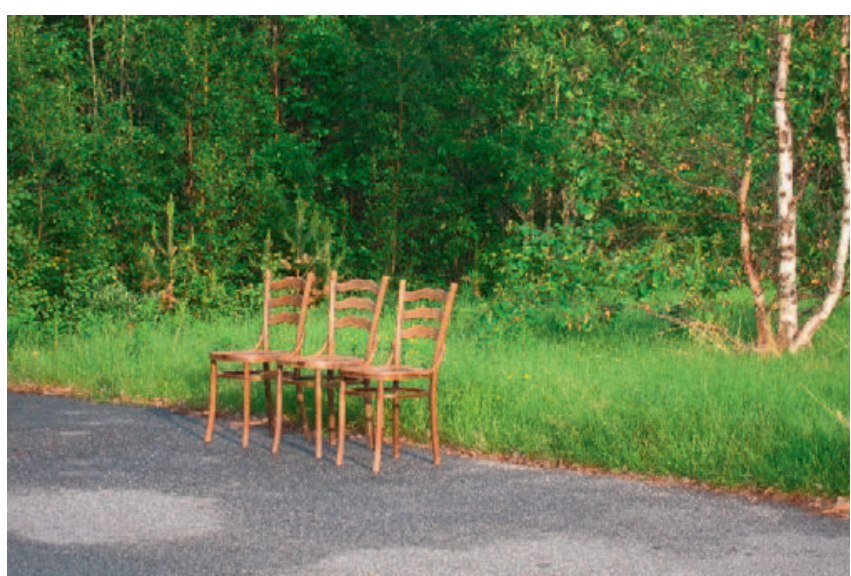

Abb. 10. Holzstühle vor Moor-Birke (Betula pubescens EHrH.), Teil 2, Norwegen (Copyright: Barbara M. Wick).

So kann die Hypothese erwogen werden, dass beispielsweise die weisse Farbe des Stammes schon vor der Kenntnis der medizinischen Wirkungen der Birke deren Symbolik (Reinheit, Neuanfang, Gutes) geprägt hat.

Eine zukunftsweisende pharmakologische Forschung könnte den aufgezeigten Symbolbezug bestätigen. Hier sei nochmals auf die weisse Farbe verwiesen: Der weisse Rinden-Farbstoff Betulin erweist sich in der aktuellen Forschung als potentes Arzneimittel gegen Bakterien, $\mathrm{Vi}$ ren, Pilze sowie Haut- und Tumorkrankheiten und könnte als «Purifikator»/«Reiniger» gesehen werden.

\section{Fazit und Ausblick}

Die Birke ist wirksam. Bei vielen Völkern sowie in der Volks- und Naturheilkunde und Phytotherapie sind die Heilwirkungen der Birke seit Langem bekannt und geschätzt.

Nach der Birken-Erkundungsreise ist abschliessend ein Ausblick angesagt:

Die aktuelle medizinische Forschung kann viele der positiven Eigenschaften belegen, sodass die pharmakologische Forschung und Industrie grosse Hoffnungen darauf setzen, bald neue Arzneimittel für die Bereiche Dermatologie, Infektiologie, Onkologie, Endokrinologie und Kardiologie entwickeln zu können.

Trotz vielversprechender Resultate gilt es zu bedenken, dass Monosubstanzen (natürliche oder synthetische) das multivalente und breite Wirkungsspektrum der traditionell angewendeten Vielstoffgemische nicht erreichen können.

Weiterer Forschungsbedarf würde nach Ansicht der Autorin bezüglich einer möglichen Wirkung der Birke gegen Depressionen bestehen [24, S. 65]. Psychische und psychosomatische Krankheiten sind mit einer hohen Morbidität und Mortalität und einer weltweit hohen Prä- 
valenz assoziiert. Trotz verschiedener Medikamentenklassen und intensiver pharmakologischer Forschung finden sich beispielsweise bei den Antidepressiva derzeit ausser dem Johanniskraut (Hypericum perforatum) wenig bahnbrechende, effektive, verträgliche und nebenwirkungsarme Psychopharmaka.

Phytopharmaka könnten effektive Alternativen in der Behandlung von psychischen Krankheiten inklusive Depressionen darstellen, insbesondere wegen der besseren
Verträglichkeit, des oft neben- und interaktionsärmeren Profils und der besseren Akzeptanz [44].

Der Anblick von Birken wirkt in jedem Fall stimmungsaufhellend und ist «nebenwirkungsfrei» (Abb. 10).

\section{Disclosure Statement}

Keine Interessenkonflikte und keine Finanzierung durch Dritte.

\section{Literatur}

1 Ashburner K, McAllister HA: The Genus Betula. A Taxonomic Revision of Birches. Kew, Kew Publications, 2013.

2 www.waldzeit.ch/buch-1997/auf-den-spurender-eiszeit/ (letzter Zugriff: 09.10.2016).

3 De Cleene M, Lejeune MC: Compendium of Symbolic and Ritual Plants in Europe. Ghent, Mens \& Cultuur Uitgevers, 2002.

4 www.pflanzenforschung.de/de/journal/journalbeitrage/birken-wirken-forscher-untersuchen-die-heilende-wirkung-10197 (letzter Zugriff: 01.10.2016).

5 Ortner HA: Die Birke. Ihre Bedeutung aus interdisziplinärer Sicht. Bern, hep, 2015.

6 www.luontoportti.com/suomi/de/linnut/birkenzeisig (letzter Zugriff: 10.10.2016).

7 https://www.youtube.com/watch? $v=$ P5ULHjuBCeo von «Botanikus.de» (letzter Zugriff: 20.09.2016).

8 Laudert D: Mythos Baum. München, BLV, 2003.

9 Lauber K, Wagner G: Flora Helvetica. Bern, Haupt, 1998.

10 Widmer R: Pflanzen im Appenzellerland. Herisau, Appenzeller, 1999.

11 www.ov-gais.ch/exkursion-botanik.html (letzter Zugriff: 05.10.2016).

12 www.iceman.it/wp-content/uploads/2016/09/ Dauerausstellung.pdf (letzter Zugriff: 05.08. 2016).

13 Fischer-Rizzi S: Blätter von Bäumen. Heilkraft und Mythos einheimischer Bäume. Aarau, AT, 2007.

14 www.waldwissen.net/wald/baeume_waldpflanzen/laub/wsl_haengebirke/index_DE (letzter Zugriff: 03.10.2016).

15 www.nordicfoodlab.org/blog/2015/11/21/treebark (letzter Zugriff: 20.09.2016).
16 www.birchwater.ch (letzter Zugriff: 21.09.2016)

17 Madaus G: Lehrbuch der biologischen Heilmittel. Abteilung 1: Heilpflanzen. Leipzig, Thieme, 1938.

18 de Haller A: Histoire des plantes Suisses ou Matière médicale et l'usage économique des plantes. Tome II. Berne, Société Typographique, 1791.

19 Hahnemann S: Apothekerlexikon. Zweiten Theils zweite Abtheilung. Leipzig, Siegfried Lebrecht Crusius, 1799.

20 Höchstetter F: Grosses, illustriertes Kräuterbuch. Reutlingen, Ensslin, 1882.

21 Oertel A, Bauer E, Rettich E: HeilpflanzenTaschenbuch. Basel, Rettich, 1909.

22 www.deutschestextarchiv.de/book/view/goethe faust01_1808?p=263 (letzter Zugriff: 05.10. 2016).

23 Sigrist A: Appenzeller Kräuterapotheke. Herisau, Appenzeller, 1997.

24 Strassmann R: Baumheilkunde. Aarau, AT, 2006.

25 Weiss RF: Das grosse Kräuterheilbuch. Olten, Walter, 1974.

26 Stammel HJ: Die Apotheke Manitous. Das medizinische Wissen der Indianer und ihre Heilpflanzen. Reinbek, Wunderlich, 1986.

27 www.kup.at/db/phytokodex/datenblatt/Birkenblatt.html (letzter Zugriff: 06.09.2016).

28 www.pharmawiki.ch/wiki/index.php?wiki=Birk e\&search=betula\#bottom (letzter Zugriff: 15.09. 2016).

29 www.ema.europa.eu/docs/de_DE/document library/EPAR_-_Product_Information/human/ 003938/WC500201153.pdf (letzter Zugriff: 29.08.2016).
30 https://www.thieme-connect.com/products/ ejournals/abstract/10.1055/s-0036-1584450 (letzter Zugriff: 19.09.2016).

31 www.morningstar.co.uk/uk/news AN_1465466135547479500/amryt-pharmaplans-trials-of-episalvan-product-by-endof-2016.aspx (letzter Zugriff: 12.10.16).

32 https://www.ncbi.nlm.nih.gov/pubmed (letzter Zugriff: 19.07.2016).

33 www.pflanzenforschung.de/de/journal/journalbeitrage/gesundheit-aus-dem-birkenwald-1119/ (letzter Zugriff: 02.10.2016).

34 www.cell.com/cell-metabolism/abstract/S15504131(10)00446-8 (letzter Zugriff: 27.09. 2016).

35 https://www.ncbi.nlm.nih.gov/pubmed/ 19367418.

36 www.aphios.com/products/research-chemicals/betulinic-acid.html.

37 www.birkenwein.ch/birke.php (letzter Zugriff: 21.06.2016).

38 www.herballegacy.com/Birch_History.html (letzter Zugriff: 27.09.2016).

39 www.rp-online.de/nrw/panorama/in-diesemmaibaum-jahr-ist-alles-anders-aid-1.5939968 (letzter Zugriff: 05.10.2016).

40 Lonitzer A: Vollständiges Kräuter-Buch. Augsburg, Joseph-Wolffsche Buchhandlung, 1783.

41 https://pwseifert.wordpress.com/2014/09/29/ tarkowskij-und-die-frauen/ (letzter Zugriff: 10.10.2016).

42 http://vogt-la.com/de/projekt/expo02 (letzter Zugriff: 05.10.2016).

43 http://vogt-la.com/de/projekt/hotel-greulich (letzter Zugriff: 10.10.2016).

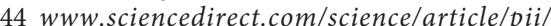
S0308814612014306 (letzter Zugriff: 12.10. 2016). 